\title{
Aproximación al análisis coste-beneficio entre empleo con apoyo y centros especiales de empleo mediante simulación comparativa con 24 trabajadores
}

\section{Approach to cost-benefit analysis between supported employment and special employment centers through comparative simulation with 24 workers}

\section{Resumen}

El presente trabajo plantea una aproximación al análisis costebeneficio comparando el empleo con apoyo (ECA) con el centro especial de empleo (CEE), desde la perspectiva de la persona, la empresa y la sociedad. Para ello, se realiza una simulación con una muestra de 24 trabajadores en empleo ordinario mediante ECA y se obtienen datos hipotéticos para los mismos trabajadores si estuviesen en un empleo de similares características en un CEE. Los resultados muestran que los trabajadores en ECA, trabajando las mismas horas, tienen mayores ganancias por hora que en CEE (9,22 € frente a 4,59 €). También, que el ECA genera menor carga social desde la empresa $(22,2 \mathrm{I} \%)$ que el CEE $(85,54 \%)$ y que la rentabilidad para la sociedad del ECA es mucho mayor $\left(3 \mathrm{I}_{5}, \mathrm{O} 3 \%\right)$ que la de los CEE $(83, \mathrm{I} 4 \%)$. Por ello, las conclusiones del estudio apuntan a que el empleo con apoyo es más ventajoso en términos de coste-beneficio para la persona, la empresa y la sociedad que los centros especiales de empleo.

\section{Palabras clave}

Discapacidad, coste-beneficio, empleo con apoyo, centro especial de empleo.

\begin{abstract}
This work presents a cost-benefit analysis comparing supported employment (SE) with special employment center (EEC), from an individual, corporate and society perspective. A simulation was carried out with a sample of 24 workers in regular employment by SE and hypothetical data were obtained for the same workers as if they were in a similar job in EEC. The results show that SE workers, working the same amount of hours, have higher hourly earnings than in EEC (9.22€ compared to $4.59 €)$. The $\mathrm{SE}$ also generates less social burden from the company $(22.2 \mathrm{I} \%)$ than EEC $(85.54 \%)$. The Supported Employment's payoff for society is much higher (3I $5.03 \%)$ than that of the EEC $(83.14 \%)$. Therefore, the conclusions of the study are directed towards the consideration that supported employment is more beneficial in terms of cost benefit for the individual, business and society when compared to the special employment centers.
\end{abstract}

\section{Keywords}

Disability, cost-benefit, supported employment, sheltered employment.
Francisco de Borja Jordán de Urríes Vega

<bjordan@usal.es>

Instituto Universitario de Integración en la Comunidad (INICO),

Universidad de Salamanca

Daniel de León Rodríguez

<danielbajamar@hotmail.com>

Máster Universitario en Investigación sobre Discapacidad del INICO, Universidad de Salamanca

\section{Francisco Hidalgo Gómez} <fhg1942@gmail.com>

Fundación Tutelar FUNDATUL

Salvador Martínez Cardeña <salvamar@gmail.com>

Fundación Tutelar FUNDATUL

\section{Mónica Santamaría Domínguez \\ <monica77@usal.es>}

Instituto Universitario de Integración en la Comunidad (INICO).

Universidad de Salamanca

\begin{abstract}
Para citar:
Jordán de Urríes Vega, F. B. et al. (20I4): "Aproximación al análisis coste-beneficio entre empleo con apoyo y centros especiales de empleo mediante simulación comparativa con 24 trabajadores”. Revista Española de
\end{abstract} Discapacidad, 2 (I): 33-50.

<http://dx.doi.org/IO.5569/23405 I04.02.01.02>

Fecha de recepción: ०8-OI-20I4 Fecha de aceptación: 20-05-20I4 


\section{Introducción}

Históricamente la discapacidad ha sido definida desde diferentes ámbitos, según los criterios de cada uno de los modelos o disciplinas que se han encargado de definirla. La Organización Mundial de la Salud (OMS) define la discapacidad como toda restricción o ausencia de la capacidad de realizar una actividad dentro del margen que se considera normal para un ser humano (Egea y Sarabia, 200I).

Los modelos biomédicos han quedado obsoletos, ya que consideran la discapacidad como una característica resultante de las deficiencias del individuo y no tienen en cuenta factores como la interacción persona-entorno (modelo biopsicosocial) [Egea y Sarabia, 200I; Maritza, 2005], las barreras físicas y sociales (modelo social), y las fortalezas del individuo y posibilidades de mejora de la funcionalidad cuando se le proporcionan los apoyos adecuados (modelo de calidad de vida) [Verdugo et al., Gómez y Navas, 20I3]. Por eso, las definiciones actuales optan por un enfoque multidimensional, que contempla dificultades y fortalezas que permitan identificar los apoyos que requiere la persona con discapacidad (PCD) y toman en cuenta no sólo las características de la persona y de su entorno, sino también otros factores contextuales (Maritza, 2005; Schalock, I999; Verdugo et al., 20I3). La discapacidad no se concibe como algo fijo ni dicotómico, sino como algo fluido, continuo y cambiante, que depende de las limitaciones funcionales de la persona, pero sobre todo, en gran medida, de los apoyos disponibles en su entorno (Verdugo et al., 2013).

En el marco conceptual anterior, y asumiendo los acuerdos internacionales (Naciones Unidas, 2006), a la persona con discapacidad deben ofertársele todas las oportunidades en igualdad de condiciones, favoreciendo su autodeterminación y calidad de vida, dando prioridad a las alternativas más integradoras (Jordán de Urríes y Verdugo, 20гоa). La integración laboral de las personas con discapacidad es un derecho constitucional y corresponde a los poderes públicos llevar a cabo políticas que eliminen las barreras que la limitan o dificultan (SEPE, 2OI2).

La Ley I3/I982, del 7 de abril, de Integración Social de los Minusválidos (LISMI) establece que "será finalidad primordial de la política de empleo de trabajadores minusválidos su integración en el sistema ordinario de trabajo o, en su defecto, su incorporación al sistema productivo mediante la fórmula especial de trabajo protegido".

El reciente Real Decreto Legislativo I/20I3, que aprueba el texto refundido de la Ley General de Derechos de las Personas con Discapacidad y de su Inclusión Social, señala que "las personas con discapacidad tienen derecho al trabajo, en condiciones que garanticen la aplicación de los principios de igualdad de trato y no discriminación".

La Constitución Española, en el artículo 35, "de los derechos y deberes de los ciudadanos", establece que "todos los españoles tienen el deber de trabajar y el derecho al trabajo, a la libre elección de profesión u oficio, a la promoción a través del trabajo y a una remuneración suficiente para satisfacer sus necesidades y las de su familia”.

El Real Decreto 870/2007, de 2 de julio, por el que se regula el programa de empleo con apoyo, tiene como objetivo favorecer la empleabilidad de estos grupos de trabajadores con discapacidad a través de un conjunto de actividades de orientación y acompañamiento individualizado, con el fin de realizar las tareas en condiciones similares al resto de los trabajadores.

La Convención de Naciones Unidas sobre los Derechos de las Personas con Discapacidad, en el artículo 27, reconoce su derecho "a trabajar, en igualdad de condiciones que las demás; pudiendo elegir libremente su trabajo en entornos abiertos, inclusivos y accesibles" (Naciones Unidas, 2006). En esta misma línea trabaja la Organización Internacional del 
Trabajo (2008), señalando que debe respetarse la igualdad de oportunidades y trato para los trabajadores, no considerándose discriminatorias las medidas encaminadas a lograr la igualdad de oportunidades (OIT, 2008).

Fruto de la LISMI y de la posterior legislación que la desarrolló, se definen diferentes modalidades de empleo para la integración de las personas con discapacidad: el empleo protegido y el empleo ordinario (Laloma, 2007). El desarrollo de los centros especiales de empleo (CEE) tiene su origen en el establecimiento de la LISMI, pero más recientemente el Real Decreto Legislativo I/20I3 determina que "son aquellos cuyo objetivo principal es el de realizar una actividad productiva de bienes o de servicios, participando regularmente en las operaciones del mercado, y tienen como finalidad el asegurar un empleo remunerado para las personas con discapacidad; a la vez que son un medio de inclusión del mayor número de estas personas en el régimen de empleo ordinario". Los CEE son, por tanto, empresas que cuentan en sus plantillas con un porcentaje de PCD no menor al $70 \%$ del total y han sido registradas en el registro correspondiente. Reúne las mismas características que el empleo ordinario, con el objetivo de la contratación de PCD que, por razón de la naturaleza o de sus minusvalías, no puedan, provisional o definitivamente, ejercer una actividad laboral en las condiciones habituales. Tiene como objetivo ser una vía de acceso al empleo ordinario (Jordán de Urríes y Verdugo, 20Iob, 20I I y 20I2; Laloma, 2007).

Por otro lado, el empleo con apoyo (ECA) es una modalidad de empleo que surge en los años ochenta en los EE. UU. como una alternativa al empleo protegido, para la inserción laboral de las PCD (Beyer y Kilsby, I998; Laloma, 2007; Shearn et al., 2000). Según la redefinición de 1998 de las Enmiendas de la Ley de Rehabilitación de I986, el ECA estadounidense es un empleo en ambientes integrados de trabajo para personas que nunca han tenido un empleo competitivo, o para aquellas que lo han tenido de forma intermitente o lo han visto interrumpido como resultado de una discapacidad significativa, quienes, a causa de una discapacidad, necesitan servicios de empleo con apoyo. En España, el Real Decreto 870/2007 y el Real Decreto Legislativo I/20I 3 definen el ECA como "el conjunto de acciones de orientación y acompañamiento individualizado en el puesto de trabajo, prestadas por preparadores laborales especializados, que tienen por objeto facilitar la adaptación social y laboral de trabajadores con discapacidad con especiales dificultades de inserción laboral en empresas del mercado ordinario de trabajo en condiciones similares al resto de los trabajadores que desempeñan puestos equivalentes”. El ECA utiliza la modalidad de colocar y entrenar (place then train), favoreciendo la integración laboral de la PCD gracias al apoyo directo en el puesto de trabajo (Jordán de Urríes, 20I I; Laloma, 2007).

\section{Análisis coste-beneficio en empleo con apoyo y protegido}

En España, no son muchos los autores que realizan aproximaciones a la cuestión del empleo y la discapacidad desde perspectivas económicas, pero sí que podemos encontrar algunos núcleos de investigación que realizan aportaciones relevantes en este sentido, como Malo (2003), Retolaza (20I2), Retolaza y Ramos (2005), Rodríguez Álvarez (20I2) y Rodríguez Álvarez et al. (2OI2).

En el ámbito internacional, durante muchos años ha habido un debate entre el ECA y el empleo protegido (Cimera, 20 I Ia). Los análisis coste-beneficio proporcionan información sobre los costes de los servicios en relación a los beneficios que de éstos se derivan, para poder así tomar decisiones al comparar entre dos opciones de servicios para un objetivo similar (Beyer y Kilsby, I998; Cimera, 2006a, 2007a, 2007b; Narrillos, 2010; Shearn et al., 2000). Estos análisis son, por tanto, utilizados para aportar datos monetarios y permitirnos conocer qué opción es la más rentable, es decir, supone un mayor beneficio en relación al gasto realizado 
(Beyer y Kilsby, I998; Cimera, 2010, 2007a, 2007b; Shearn et al., 2000), siendo importante que ambas opciones sean equivalentes, o la comparación perdería la utilidad (Cimera, 2007a, 2007b). El problema de estos análisis es la mutabilidad, ya que en el momento que cambie una de las variables contempladas (subvenciones, aumentos salariales, cambio del valor monetario, etc.), los resultados no serían los mismos (Cimera, 20I 2a); y también, que sólo hacen referencia a cosas cuantificables y no conceptualizan adecuadamente los beneficios no tangibles ni monetarios, como los emocionales, sociales u otros (Beyer y Kilsby, I998; Cimera, 2007a, 2007b; Cimera y Burgess, 20II).

Para analizar el coste-beneficio, los estudios tienen en cuenta múltiples variables, como datos demográficos, tipo de discapacidad, tipo de empleo, horas de trabajo, información salarial y gastos (Beyer, 20I2; Beyer y Kilsby, I998; Beyer, Kilsby, y Shearn, I999; Cimera, 2007a, 2008, 2009a, 2009b, 20I0, 20IIa, 20I Ib; Cimera y Burgess, 20II). El Cuadro I nos muestra diferentes tipos de variables utilizadas en análisis coste-beneficio, dependiendo de la perspectiva de análisis.

A pesar de algunas críticas que se han podido realizar hacia el ECA (Cimera, 2006b), muchos han sido los estudios que han sostenido que proporciona importantes beneficios desde la perspectiva de la persona, la empresa o la sociedad, diluyéndose los costes, al igual que la figura de apoyo, a medida que pasa el tiempo (Beyer y Kilsby, I998; Beyer et al., I999; Cimera, 2007a, 2007b, 2008, 201 2a). Podemos encontrar evidencias de resultados (Beyer, 20I2; Beyer et

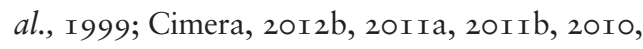
2009a, 2008, 2007a, 2006a; Cimera y Burgess, 20I I; Shearn et al., 2000) sobre los beneficios del ECA y sus mejores resultados respecto al empleo protegido desde las tres perspectivas de análisis citadas: persona, empresa y sociedad.

\subsection{Perspectiva de la persona}

Existen evidencias que ponen de manifiesto que las PCD intelectual se benefician de trabajar en la comunidad, ya que por cada dólar estadounidense al que renuncian por otras vías de ingresos cuando llegan al empleo competitivo perciben $4,20 \$$ y alcanzan beneficios netos mensuales de 475,35\$ (Cimera, 20Ioa). Lo mismo ocurre entre personas con autismo, que perciben 5,28 \$ por cada dólar renunciado y alcanzan beneficios netos mensuales de 643,20\$ (Cimera y Burgess, 20II).

Contra la creencia de que el paso por el empleo protegido prepara mejor para el acceso al empleo ordinario, cuando se compara a las personas que acceden al ECA desde empleo protegido con las que no, se percibe que las primeras tienen menores ganancias: I I 8,35\$ frente a I37,20\$(Cimera, 201 Ia). Además, cuando acceden a un empleo, las personas que anteriormente han trabajado en otro empleo mediante ECA consiguen mejor salario y trabajan más horas que las que acceden al empleo por primera vez (Cimera, 20I 2b).

En la comparación entre ECA y empleo protegido respecto al salario mensual, el ECA aporta mayores beneficios al mes, con 390,96 \$ de salario medio frente a 164,79 \$ en empleo protegido (Cimera, 20 I Ib). Además, independientemente de la discapacidad, la persona se beneficia de trabajar en ECA más que si lo hace en empleo protegido, aunque las ganancias no le permitan en ningún caso salir de un bajo nivel de ingresos (Cimera, 20I 2a).

\subsection{Perspectiva de la empresa}

$\mathrm{Al}$ analizar desde esta perspectiva, encontramos el no poco alentador dato de que las PCD reportan más beneficios al empresario que las personas sin discapacidad si nos atenemos a que las personas con discapacidad permanecen más tiempo en sus empleos, presentan menor absentismo y llegan menos veces tarde al trabajo (Cimera, 2009b).

La evolución de los costes globales de la empresa tiende al descenso en el ECA, mientras que aumenta en el empleo protegido (Cimera, 2008). Los datos ponen de manifiesto cómo durante un 


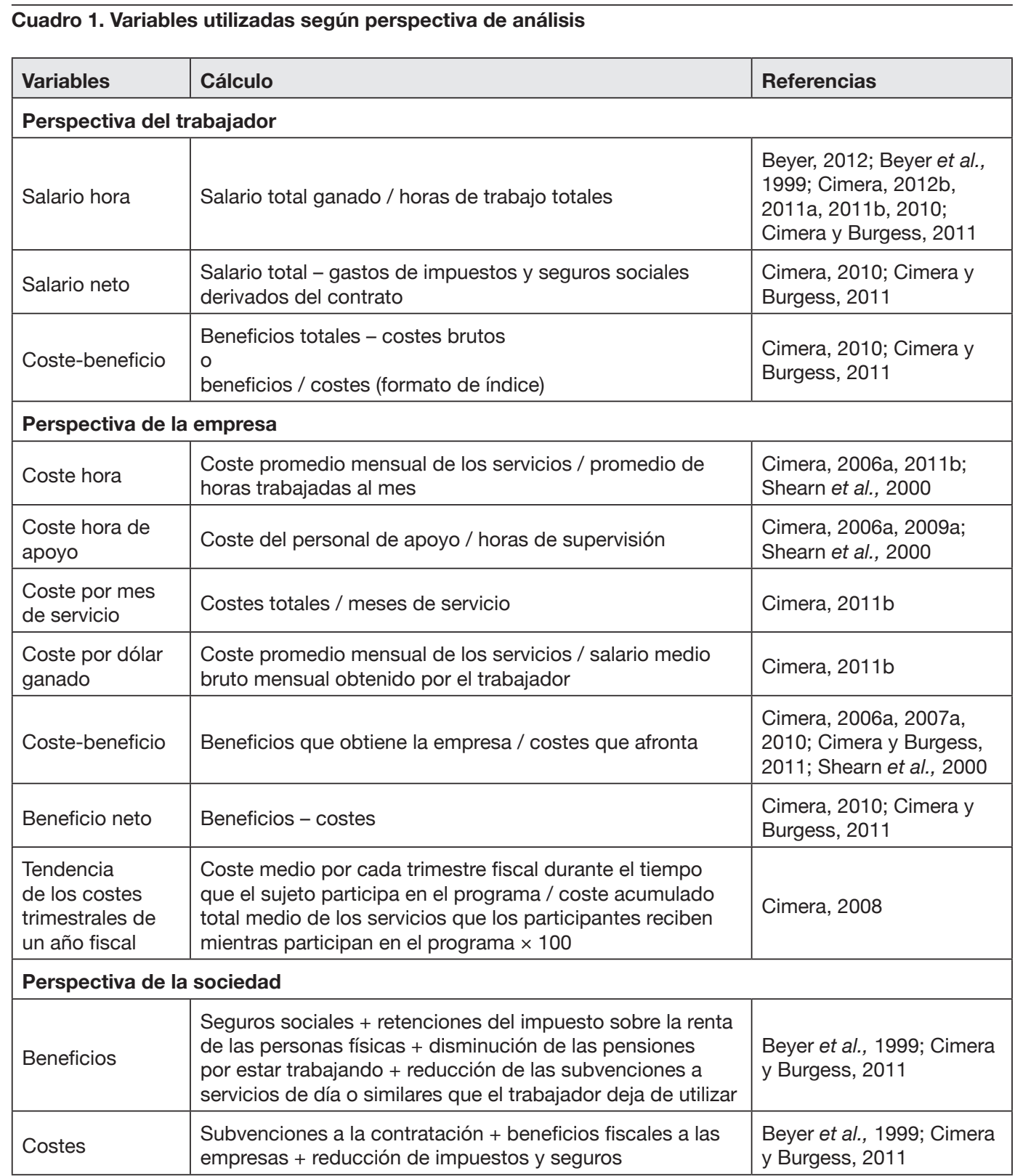

Fuente: Elaboración propia.

periodo analizado de tres años, en los primeros tres trimestres el ECA genera el 36,6\% del total acumulativo de sus costes, reduciéndose a un $6,6 \%$ en los tres últimos trimestres. Sin embargo, en el mismo periodo, el empleo protegido genera en los primeros tres trimestres el I $4,4 \%$ de sus costes acumulativos, que alcanzan un $\mathbf{2 7 , 5} \%$ en los últimos tres meses.
Para la empresa (Cimera, 20I2b), el coste del apoyo a las personas con experiencia en ECA que pasan de un empleo en la comunidad a otro (5.1 $32,46 \$)$ es menor que el de las personas que se emplean por primera vez en un empleo en la comunidad $(5 \cdot 726,39 \$)$. Cabe señalar aquí los resultados de un trabajo anterior (Cimera, 20I Ib). En ellos es interesante apreciar la 
comparación entre ECA y empleo protegido, encontrándose que las personas en ECA tienen menor coste por mes de servicio $(5$ I $2,79 \$)$ que aquéllas en empleo protegido $(586,28 \$)$ y generan menos costes netos (22.406\$) en ECA que en empleo protegido $(45.840 \$)$; e incluso trabajando menos horas, los trabajadores en ECA son más rentables en coste por hora de trabajo (I I, I 5 \$) que los de empleo protegido $\left(\mathrm{I}_{5}, \mathrm{O}_{4} \$\right)$.

En cuanto al análisis coste-tendencia, se ha observado que la tendencia es inversamente proporcional: mientras que en ECA los costes acumulados comienzan en un I I, $78 \%$ y descienden al I, I $3 \%$ tras I I cuatrimestres, en empleo protegido empiezan en un $6,80 \%$ y alcanzan el I0,96\% en el mismo periodo (Cimera, 2008). Cimera aporta varias explicaciones posibles para estos resultados. Una es que los empleados en centros de empleo protegido reciben más servicios cuanto más tiempo permanecen en ellos. Otra posibilidad es que cuanto más tiempo se mantiene un trabajador en empleo protegido, tiende a tener más horas de trabajo y, por lo tanto, genera más costes relacionados con la mayor frecuencia de supervisión del trabajo. Esta interpretación, según Cimera, se ve corroborada por otros autores, que encuentran que cuanto más tiempo se permanece en empleo protegido, menos probable es que el trabajador pase a un empleo ordinario en la comunidad.

\subsection{Perspectiva de la sociedad}

Finalmente -aunque no menos relevante-, desde la perspectiva de la sociedad, es decir del contribuyente, el empleo con apoyo es más coste-eficiente para las PCD intelectual que el empleo protegido. Por cada persona que se encuentra en empleo protegido, se puede amortizar a tres personas dentro de la comunidad en empleo con apoyo. Los resultados del coste acumulativo medio entre empleo con apoyo y empleo protegido son significativamente diferentes durante su ciclo de trabajo $(6.6$ I $8,76 \$$ frente a I9.388,04\$) [Cimera, 2008].
Cimera pone de manifiesto que la PCD en ECA es rentable en el análisis coste-beneficio, ya que por cada dólar que la sociedad invierte, la persona devuelve un promedio de $1,46 \$$ (Cimera, 20I 2a). No ocurre lo mismo en los datos que aportan Beyer y sus colaboradores (I999), donde se aprecia que el ECA presenta un retorno al contribuyente inferior a lo que la sociedad invierte, ya que la sociedad recibe de media solamente 0,43 peniques por cada libra esterlina invertida. Sin embargo los datos de Beyer indican que este retorno evoluciona positivamente con los años, apreciándose un aumento desde los 7 peniques por libra invertida a los 54 peniques por libra invertida en aquellos programas que mantienen sus servicios durante 6 o más años (Beyer et al., I999).

\section{Problema y objetivo de la investigación}

El desempeño de un trabajo es un objetivo prioritario que tienen todas las personas y una vía de acceso a la vida adulta, permite acceder a elementos indispensables para la vida plenamente integrada en sociedad y proporciona a la persona cierto estatus social. Estos objetivos no son diferentes en las PCD.

En el periodo de crisis que sufrimos, el acceso al mercado laboral cada vez se está complicando en mayor medida, y si antes era difícil para la PCD, en estos momentos lo está siendo aún más. El Instituto Nacional de Estadística indicaba en 20I I que la evolución de la tasa de paro en el colectivo de personas con discapacidad había evolucionado en los últimos tres años de un $2 \mathrm{I}, 8 \%$ en 2009 , a un $23,3 \%$ en 2010 , llegando al $26,9 \%$ en $20 \mathrm{II}$, mostrando una evolución peor que entre las personas sin discapacidad, que pasaron de un I $8, \mathrm{I} \%$ a un $20, \mathrm{I} \%$ y un $2 \mathrm{I}, 7 \%$, respectivamente, en esos mismos años (INE, 2OII). Es previsible que la situación en las personas con discapacidad sea aun peor hoy día, con la tasa de paro del $25,93 \%$ entre la población general en el primer trimestre de 20I4 (INE, 20I4). 
Por ello, cobran una mayor importancia aun si cabe los estudios de coste-beneficio, que nos permiten decantarnos por la opción más rentable para trabajadores, empresas y sociedad. Estudios realizados en otros países, principalmente en los Estados Unidos, muestran que el ECA ofrecen mayor rentabilidad que los CEE (Cimera, 2006a, 2007a, 2008, 2009b, 20I Ia, 20I2b; Shearn et al., 2000). La situación del empleo protegido es cualitativamente diferente en España, y nuestros CEE difieren de los talleres protegidos que funcionan en Estados Unidos. En nuestro país, carecemos de análisis comparativos de ambas realidades desde este enfoque. Trabajando, pues, en esta línea, el presente estudio trata de realizar una aproximación al análisis coste-beneficio entre empleo con apoyo y CEE en España, desde las perspectivas del propio trabajador, la empresa y la sociedad. Para ello, desarrolla una simulación comparativa con 24 trabajadores en puestos de trabajo en la comunidad mediante empleo con apoyo, con los cuales se realiza una simulación de costes en una hipotética situación laboral similar en un CEE.

El objetivo es, por tanto, analizar los costes en una misma situación hipotética de una PCD en un ECA y en un CEE a través de una simulación. Dado el carácter descriptivo, no pretendemos establecer ningún tipo de causa-efecto entre variables, simplemente mostrar las diferencias existentes en función del grupo de análisis. Los cálculos se realizaron desde la perspectiva del trabajador (cantidad neta que percibe y sueldo/ hora), la empresa (coste neto, coste/hora y porcentaje de carga social) y la sociedad (coste para la sociedad, coste/hora, rentabilidad y rentabilidad por euro invertido).

El estudio se realizó entre marzo de 20 I 2 y febrero de 2013, con nóminas correspondientes a mayo, junio y julio de 20I2. Los cálculos de las ayudas a las contrataciones se hacen en relación a las vigentes en ese momento.

\section{Procedimiento y selección de la muestra}

Para la consecución de la muestra, se contactó con la Fundación Tutelar y de Asistencia Personal Fundatul y se les explicó cuál era el objetivo del estudio. Cuanto la fundación decidió participar en él, nos proporcionó los datos necesarios, con el consentimiento de los usuarios.

La población objeto de estudio fueron 24 PCD, con edades comprendidas entre I 8 y 65 años, de ambos sexos, que se encontraban trabajando mediante ECA en un puesto de trabajo en empresa ordinaria. Con cada uno de ellos se realizó una simulación de ubicación en un CEE, que nos proporcionó datos ficticios para comparar. Los criterios de inclusión fueron: ser una PCD, tener una edad de entre I 8 y 65 años, estar empleado y que la fundación dispusiera de una copia de la nómina.

El muestreo utilizado fue no probabilístico incidental, y todos los trabajadores que atendían a los criterios de selección y habían proporcionado los datos necesarios para el estudio podían formar parte de él. Este tipo de muestreo, si bien muestra claras limitaciones en la generalización de resultados, es, sin embargo, muy comúnmente utilizado en el ámbito de la discapacidad, por la dificultad de selección de muestras probabilísticas.

La muestra finalmente seleccionada para el estudio estaba compuesta por 24 PCD. La mitad eran varones, con una media de edad de 36,50 años; y la otra mitad, mujeres, con una media de edad de 32,83 años; la edad media total de los participantes era de 34,67 años. En la Tabla I, se muestran los descriptivos de frecuencia y porcentaje válido para las variables sexo, discapacidad, tipo de discapacidad, edad y empleo. Todas las personas participantes se encontraban trabajando en empresas ordinarias mediante empleo con apoyo, y para cada una se simuló la misma situación de empleo en centro especial de empleo. 
Tabla 1. Descriptivos de frecuencia y porcentaje válido de las variables demográficas

\begin{tabular}{|l|l|c|c|}
\hline \multicolumn{2}{|c|}{} & Frecuencia & \% válido \\
\hline \multirow{4}{*}{ Sexo } & Hombre & 12 & 50,0 \\
\cline { 2 - 4 } & Mujer & 12 & 50,0 \\
\hline \multirow{4}{*}{ Grado de discapacidad (\%) } & $34-44$ & 13 & 54,2 \\
\cline { 2 - 4 } & $46-64$ & 4 & 16,7 \\
\cline { 2 - 4 } & $>65$ & 7 & 29,2 \\
\hline \multirow{3}{*}{ Tipo discapacidad } & físico & 8 & 33,3 \\
\cline { 2 - 4 } & intelectual & 10 & 41,7 \\
\cline { 2 - 4 } & sensorial & 6 & 25,0 \\
\hline \multirow{3}{*}{ Edad (años) } & $<45$ & 20 & 83,3 \\
\cline { 2 - 4 } & $>45$ & 4 & 16,7 \\
\hline \multirow{2}{*}{ Tipo de contrato } & indefinido & 10 & 41,7 \\
\cline { 2 - 4 } & eventual & 14 & 58,3 \\
\hline
\end{tabular}

Fuente: Elaboración propia.

\section{Descripción de las variables y procedimiento de análisis}

Para el desarrollo del estudio, se conceptualizó la modalidad de empleo (ECA y CEE) como variable independiente, $\mathrm{y}$ como variable dependiente genérica, el análisis coste-beneficio. Este último se afronta desde tres perspectivas (trabajador, empresa y sociedad), en cada una de las cuales se analizan variables diferentes, que se explican y operativizan con detalle más adelante.

El paquete estadístico SPSS se utilizó para hacer un análisis descriptivo de las variables demográficas, para conocer cómo son las situaciones de la muestra con la que tratamos. También se utilizó una hoja de cálculo Excel para hacer los análisis necesarios desde cada una de las tres perspectivas mencionadas.

Las 24 personas de la muestra, como se ha indicado, desarrollaban un empleo ordinario con apoyo y, para poder comparar a las mismas personas en una hipotética situación en CEE, se realizó con cada una una simulación con un mismo tipo de contrato (en número de horas y tipo de trabajo desarrollado) en CEE, atendiendo a las subvenciones y bonificaciones vigentes en ese momento. Los tipos y cuantías de ayudas disponibles en julio de 2012 para la contratación de personas con discapacidad en empresas ordinaria y aquellas a las que pueden acceder los CEE se describen en los Cuadros 2 y 3, basados en la información de la Guía laboral del Ministerio de Empleo y Seguridad Social (2OI2) ${ }^{\mathrm{I}}$.

Hay que señalar que, para los cálculos de subvenciones por contrato indefinido, tanto en empresa ordinaria como en CEE, dada la obligatoriedad de mantener el contrato por tres años, las cantidades se dividieron entre tres por considerarse el periodo de amortización de las subvenciones de tres años. Indicamos también que, para el cálculo del número de horas y sueldo en los contratos de empresa ordinaria, se aplicó en todos los casos el convenio de hostelería, por ser el que más representaba al conjunto de la muestra seleccionada. En la simulación de contratos en CEE, el convenio

I. Puede consultarse también el trabajo de Rodríguez Álvarez (2OI2). 
Cuadro 2. Ayudas a la contratación de personas con discapacidad en empresa ordinaria

\section{Contratación indefinida}

- Subvención por contrato a tiempo completo:

- 3.907,00 € (que se reducen proporcionalmente en el tiempo parcial).

- Bonificaciones de la cuota de la Seguridad Social durante la vigencia del contrato:

- Menor de 45 años.

- General: 4.500,00€.

- Discapacidad severa*: 5.100,00€.

- Mujeres:

- General: 5.350,00€

- Discapacidad severa*: 5.950,00€.

- Mayor de 45 años:

- General: 5.700.00€.

- Discapacidad severa*: 6.300,00€.

- Subvención de una sola vez para adaptación de puestos de trabajo.

- Hasta 901,52€.

\section{Contratación temporal para el fomento del} empleo

- En general.

- Varones.

- Menor de 45 años: 3.500,00€.

- Mayor de 45 años: 4.100,00€.

- Mujeres.

- Menor de 45 años: 4.100,00€.

- Mayor de 45 años: 4.700,00€.

- Discapacidad severa.

- Varones.

- Menor de 45 años: 4.100,00€.

- Mayor de 45 años: 4.700,00€.

- Mujeres.

- Menor de 45 años: 4.700,00€.

- Mayor de 45 años: 5.350,00€.

* Personas con parálisis cerebral, enfermedad mental o discapacidad intelectual igual o superior al $33 \%$ y discapacidad física o sensorial igual o superior al $65 \%$. Fuente: Elaboración propia a partir de Ministerio de Empleo y Seguridad Social (20I2).
Cuadro 3. Ayudas a la contratación de personas con discapacidad en CEE

\section{Ayudas a los proyectos generadores de empleo}

- Tipo:

- Para asistencia técnica.

- Para sufragar la inversión fija en proyectos de interés social.

- Para sufragar parcialmente los intereses de los préstamos que obtengan de entidades de crédito, públicas o privadas, que tengan suscrito convenio.

- Cuantía:

- Las subvenciones serán, en su conjunto, de $12.020,24 €$ por puesto de trabajo creado con carácter estable si el centro especial de empleo supera el $90 \%$ de trabajadores con discapacidad respecto al total de su plantilla.

- La cuantía de la subvención por puesto de trabajo creado de carácter estable será de $9.015,18 €$ si el número de trabajadores con discapacidad del centro especial de empleo está comprendido entre el $70 \%$ y el $90 \%$ del total de la plantilla.

Ayudas para el mantenimiento de puestos de trabajo en centros especiales de empleo

- Tipo:

- Subvención del coste salarial

- Bonificación del $100 \%$ de la cuota empresarial a la Seguridad Social, incluidas las de accidentes de trabajo y enfermedad profesional, así como las cuotas de recaudación conjunta por contratos indefinidos o temporales, incluidos los contratos formativos.

- Subvenciones del coste salarial correspondiente al puesto de trabajo ocupado por personas con discapacidad que realicen una jornada de trabajo laboral normal y que estén de alta en la Seguridad Social, por un importe del $50 \%$ del salario mínimo interprofesional.

- Subvención para la adaptación de los puestos de trabajo y eliminación de barreras arquitectónicas:

- Cuantía no superior a $1.803,04 €$ por puesto de trabajo, sin que en ningún caso rebase el coste real que, al efecto, se justifique.

- Subvención, por una sola vez, destinada a equilibrar y sanear financieramente los centros especiales de empleo.

- Subvención dirigida a equilibrar el presupuesto de aquellos centros especiales de empleo que carezcan de ánimo de lucro y sean de utilidad pública e imprescindibilidad.

Fuente: Elaboración propia a partir de Ministerio de Empleo y Seguridad Social (20I2). 
aplicado es el genérico de CEE, por no poderse aplicar otro. También es reseñable que a cada caso se le aplicó la modulación correspondiente a edad, sexo y grado de discapacidad. De igual manera, se ha tenido en cuenta si el tipo de contrato era indefinido o temporal.

No hemos considerado procedente computar a ningún efecto los costes de los preparadores laborales en ECA, ni tampoco los del personal de las unidades de apoyo a la actividad profesional en los CEE. Por un lado, porque consideramos que esos costes no refieren directamente a la contratación, sino a la preparación del trabajador. Por otro, no parece adecuado aventurar cálculos cuando las ratios reales de usuario por preparador laboral en ECA superan en muchos casos las establecidas en el Real Decreto 870/2007, por causa de la escasez de recursos y la necesidad de mantener los servicios. El Informe contrataciones de empleo con apoyo realizado por la Asociación Española de Empleo con Apoyo (20I2) pone de manifiesto que un $76 \%$ de los servicios de ECA se financian a través de convocatorias de financiación pública, y que, de ellos, el $80 \%$ utiliza fuentes diferentes al citado Real Decreto, lo que hace extremadamente difícil cuantificar la financiación por usuario en función de los datos genéricos de los que disponíamos para el estudio, extraídos del análisis de los contratos.

Una vez recabados los datos, se realizaron comparaciones en las variables anteriormente descritas y desde las perspectivas del trabajador, la empresa y la sociedad. Para el cálculo de los resultados, en cada variable analizada se optó por dos métodos diferentes. En un método, se decidió obtener los datos tomando a la muestra en conjunto, calculando los sumatorios $\left(\sum\right)$ de las cantidades anuales totales para el conjunto de la muestra en cada una de las variables (por ejemplo, la suma de sueldos netos de todos los trabajadores de la muestra y la suma de horas de trabajo) y realizando el cálculo con esos sumatorios (para averiguar, por ejemplo, el sueldo neto por hora). En el otro método, se optó por calcular el dato concreto para cada trabajador (sueldo neto de cada trabajador dividido entre sus horas de trabajo, para obtener el sueldo neto hora) y posteriormente calcular el dato medio analizado $(\bar{x})$ [realizando la media para la muestra de todos los sueldos netos/hora]. A continuación se presentan los resultados obtenidos desde las tres perspectivas de análisis.

\subsection{Variables en la perspectiva del trabajador}

El sueldo neto es la cantidad que percibe el trabajador por el trabajo que realiza. Se calcula restando al salario bruto del trabajador las retenciones de los seguros sociales y del impuesto sobre la renta de las personas físicas (IRPF). Los trabajadores en CEE no pagarían retenciones de IRPF, por no alcanzar sus salarios el nivel impositivo requerido. Esta variable no es comparable en sí misma, pues depende de la jornada de trabajo real, pero nos sirve para el cálculo del sueldo hora.

El sueldo neto hora es la cantidad neta que percibe el trabajador por una hora de trabajo. Se obtiene mediante un cociente entre el salario neto anual del trabajador y las horas totales de trabajo.

\subsection{Variables en la perspectiva de la empresa}

El coste neto es la cantidad que le cuesta a la empresa un trabajador. Se calcula sumando inicialmente los costes que tiene la empresa, que son el salario y los seguros sociales en la empresa ordinaria y sólo el salario en el CEE, y posteriormente restando a lo obtenido las subvenciones que recibe la empresa, que en la ordinaria por medio de ECA son las de los seguros sociales, la subvención de contrato indefinido y la deducción de impuestos, y que en el caso del CEE además incluyen las ayudas de apoyo técnico y del salario mínimo. Estas cantidades en los cálculos mensuales se calculan de manera proporcional sobre el total que se recibe.

El coste neto hora es el importe que paga la empresa por cada hora de trabajo de la persona. Se calcula mediante un cociente entre el coste neto anual para la empresa y las horas anuales de un trabajador. 
El porcentaje de carga social es la relación entre lo que la sociedad aporta para ese contrato y los costes de éste. Se computa mediante una división entre las bonificaciones de la Administración y los costes de la empresa.

\subsection{Variables en la perspectiva de la sociedad}

El coste neto sociedad es el gasto que tiene la sociedad por que las personas se encuentren empleadas. En ECA, se calcula mediante una resta entre las subvenciones (seguros sociales + subvención de contrato indefinido + deducción de impuestos); en CEE además se suman el apoyo técnico, el salario mínimo y los retornos (seguros sociales de la empresa y el trabajador + IRPF). Los trabajadores en CEE no pagarían retenciones de IRPF, por no alcanzar sus salarios el nivel impositivo requerido.

El coste hora sociedad se computa mediante una división entre el coste neto para la sociedad y el total de horas de trabajo.

El porcentaje de retorno socioeconómico es la eficiencia que tiene un empleo en relación a las ayudas que recibe. Se computa mediante un cociente entre el salario neto que percibe el trabajador y los costes de la sociedad, es decir, las subvenciones que proporciona.

\section{Resultados}

\subsection{Resultados en la perspectiva del trabajador}

Respecto al sueldo neto -que no es una variable comparable, pero la necesitamos para obtener el sueldo hora-, vemos, a modo de ejemplo, que su cálculo con un trabajador de la muestra en ECA se realizaría partiendo de un salario bruto mensual de $.640 €$, al que le restaríamos $99,22 €$ de seguros sociales y otros $97,74 €$ de retención de IRPF, de forma que quedaría un salario neto de I.443,04€. Simulando su contrato en un CEE, este mismo cálculo partiría de un salario bruto mensual de $748,30 €$, establecido por ser un trabajador a jornada completa, menos 45,27€ de seguros sociales; no pagaría retenciones de IRPF, al no alcanzar su salario el nivel impositivo requerido. Por tanto, nos quedaría un salario neto de 703,03 €.

En cuanto al sueldo neto hora-que sí podemos comparar y que se describe como hemos mencionado en el apartado de variables-, sabiendo que en ECA un trabajador, a modo de ejemplo, cobra un neto anual de I7.3 I6,43 € y trabaja un total de $1.826 \mathrm{~h}$ anuales, dividiendo lo primero entre lo segundo, se obtiene un sueldo hora de 9,48€. Simulando su contrato en un CEE y trabajando las mismas horas, esta misma persona cobraría un total de 8.433,46€, lo que nos da, al hacer la división, una cantidad de $4,62 €$ por cada hora de trabajo.

Para el cálculo con el total de la muestra, se ha realizado, por un lado, un sumatorio anual de cada uno de los conceptos, que después se ha dividido entre los totales; y por otro lado, se ha calculado el dato para cada trabajador y se obtenido luego el dato medio. Podemos observar los resultados en la Tabla 2. Tenemos, por tanto, que el sueldo hora de los trabajadores en ECA asciende a 9,22€, mientras que al simular los contratos en CEE el sueldo hora llega sólo a $4,59 €$. Si utilizamos el cálculo de los sueldos netos hora de cada trabajador y realizamos la media, los datos son ligeramente más bajos, alcanzando 9,03 € en ECA y 4,53 € en CEE.

Tabla 2. Sueldo neto hora de los 24 trabajadores de la muestra en las dos modalidades de empleo

\begin{tabular}{|l|l|l|l|l|}
\hline & $\begin{array}{c}\sum \text { Sueldo } \\
\text { neto anual }\end{array}$ & $\begin{array}{c}\sum \text { Horas } \\
\text { anuales }\end{array}$ & $\begin{array}{c}\text { Sueldo } \\
\text { neto } \\
\text { hora }\end{array}$ & $\begin{array}{c}\bar{x} \text { Sueldo } \\
\text { neto } \\
\text { hora }\end{array}$ \\
\hline ECA & $304.968,54 €$ & 33.072 & $9,22 €$ & $9,03 €$ \\
\hline CEE & $151.920,42 €$ & 33.072 & $4,59 €$ & $4,53 €$ \\
\hline
\end{tabular}

Fuente: Elaboración propia. 


\subsection{Resultados en la perspectiva de la empresa}

Para realizar el cálculo del coste neto para la empresa, como podemos ver, a modo de ejemplo, con un trabajador en ECA, se suman, por un lado, los costes del salario mensual ( $.640 €)$ y de Seguridad Social $(525,62 €)$, lo que supone un total de $2.165,62 €$. Por otro lado, sumamos los ingresos de las bonificaciones en seguros sociales que recibe por ese contrato $(495,83 €)$, la subvención por contrato indefinido (I3 I,94 €) y la deducción de impuestos (I 66,67 €), lo que nos arroja un total de $794,44 €$. Si realizamos la diferencia de esas dos cantidades (costes e ingresos), obtenemos I.37I, I $8 €$ de coste neto para la empresa.

Si realizamos la simulación de este mismo trabajador en un puesto en CEE, un contrato del mismo tipo le costaría a la empresa solamente el importe del salario mensual $(748,30 €)$, ya que el CEE está exento de pagar seguros sociales. Por otro lado, los ingresos por las bonificaciones deberían sumar la subvención por contrato indefinido $(333,92 €)$, más el apoyo técnico ( Iо०€) y la subvención del salario mínimo $\left(374, \mathrm{I}_{5} €\right)$, lo que supone un total de $808,07 €$. Si hallamos la diferencia entre las cantidades obtenidas (costes e ingresos), llegamos a $-59,77 €$ de coste neto para el CEE, es decir, un beneficio neto positivo de $59,77 €$.

El coste neto hora para un trabajador en empresa ordinaria mediante ECA se calcula, a modo de ejemplo, dividiendo el coste neto anual del trabajador (I6.45 I,I I €) entre el número de horas de trabajo anuales ( $1.826 \mathrm{~h}$ ), lo que supone un coste neto 9, ОI $€$ la hora. Simulando el mismo cálculo para el mismo trabajador en un CEE con un coste neto anual de -7 I 2,20 $€$ y el mismo número de horas anuales (I.826), supone $-0,39 €$ la hora, es decir, beneficio neto de $0,27 €$ la hora.

En cuanto al porcentaje de carga social para un trabajador en empresa ordinaria mediante ECA, se calcula, a modo de ejemplo, sumando las bonificaciones que recibe la empresa $(794,44 €)$ y dividiéndolas entre los costes que tiene $(2.165,62 €)$, lo que nos da una cantidad de $0,37 €$, es decir, una carga social del $37 \%$. Realizando la simulación del mismo trabajador en un CEE, la suma de las bonificaciones ascendería a $808,07 €$, y la de los costes, a $748,30 €$, por lo que en CEE el cociente sería de $\mathrm{I}, \circ 8 €$, o lo que es lo mismo, una carga social del Io $8 \%$.

Para el cálculo con el total de la muestra, se ha realizado, por un lado, un sumatorio anual de cada uno de los conceptos, que se ha dividido entre los totales; y por otro lado, se ha calculado el dato para cada trabajador, así como el dato medio. Podemos observar los resultados en la Tabla 3. Manejando los sumatorios, obtenemos, por tanto, que el coste neto hora de los trabajadores en ECA es de $10,25 €$, mientras que al simular los contratos en CEE el coste neto hora llega solamente a $0,93 €$. Si calculamos los costes netos hora para cada trabajador y posteriormente la media de éstos, los resultados alcanzan un coste neto hora de $9,85 €$ en ECA y de $0,72 €$ en CEE.

Tabla 3. Coste neto hora y porcentaje de carga social del total de los 24 trabajadores de la muestra en las dos modalidades de empleo

\begin{tabular}{|l|c|c|c|c|}
\hline & $\sum$ Coste neto anual empresa & $\sum$ Horas anuales & Coste neto hora & $\bar{x}$ Coste neto hora \\
\hline ECA & $339.121,01 €$ & $33.072 €$ & $10,25 €$ & $9,85 €$ \\
\hline CEE & $30.886,48 €$ & $33.072 €$ & $0,93 €$ & $0,72 €$ \\
\hline & $\sum$ Bonificaciones & $\sum$ Costes & $\%$ Carga social & $\bar{x} \%$ Carga social \\
\hline ECA & $96.805,72 €$ & $435.926,73 €$ & $22,21 \%$ & $23,99 \%$ \\
\hline CEE & $182.732,23 €$ & $213.618,71 €$ & $85,54 \%$ & $89,74 \%$ \\
\hline
\end{tabular}

Fuente: Elaboración propia. 
Paralelamente, manejando los sumatorios, vemos que la carga social alcanza en ECA el 22,2I \%, mientras que en CEE asciende al $85,54 \%$. Si calculamos el porcentaje de carga social en cada trabajador y posteriormente realizamos la media de éstos, los resultados alcanzan el $23,99 \%$ en ECA y el $89,74 \%$ en CEE.

\subsection{Resultados en la perspectiva de la sociedad}

Para averiguar el coste neto sociedad, a modo de ejemplo, para una persona en empresa ordinaria mediante ECA, sumamos primero los costes para la sociedad, que provienen de la subvención por contrato indefinido ( 13 I,94 $€$ ), la deducción de impuestos ( $166,67 €$ ) y las bonificaciones de seguros sociales $(495,83 €)$, lo que supone un total de $794,44 €$. Por otro lado, calculamos los retornos, que serían la suma de los ingresos de seguros sociales $(29,79 €)$, los de cuota obrera $(99,22 €)$ y los de IRPF $(97,74 €)$, que hacen un total de $226,75 €$. Realizando la diferencia entre los costes a la sociedad y los retornos para esta persona, obtenemos un coste neto para la sociedad de 567,69€ mes.

Simulando el mismo cálculo con ese mismo trabajador en un CEE, sumaríamos como coste para la sociedad el de la subvención por contrato indefinido $(333,9 \mathrm{I} €)$, el del apoyo técnico ( $100 €)$, el de los seguros sociales $(239,83 €)$ y la bonificación de salario mínimo $\left(374, \mathrm{I}_{5} €\right)$, lo que suma un total de $\mathrm{I} .047,89 €$. Por otro lado, los retornos serían exclusivamente la cuota obrera $(45,27 €)$, ya que no pagarían retenciones de IRPF, por no alcanzar su salario el nivel impositivo requerido. Realizando la diferencia entre los costes a la sociedad y los retornos para esta persona, obtenemos un coste neto para la sociedad de I.002,62€.

El coste hora sociedad para un trabajador en empresa ordinaria mediante ECA se calcularía, a modo de ejemplo, sobre un total de I.826 $\mathrm{h}$ trabajadas al año y un coste neto anual de 6.8 I $2,33 €$, realizando el correspondiente cociente, que nos proporciona una cifra de 3,73€. Simulando la misma situación para este trabajador en un CEE que trabajara el mismo número de horas anuales ( $\mathrm{I} .826 \mathrm{~h}$ ) y con un coste neto anual de I2.03 I, $50 €$, el coste hora sería de $6,59 €$.

El porcentaje de retorno socioeconómico para un trabajador en empresa ordinaria mediante ECA se calcularía, a modo de ejemplo, realizando un cociente entre su salario neto anual (I7.3 I6,33 €) y la cantidad que proporciona en subvenciones la sociedad $(9.533,33 €)$, lo que arrojaría un resultado de I,8 I $64 €$, o lo que es lo mismo, un porcentaje de retorno socioeconómico del I 8I, $64 \%$. Simulando la misma situación para este trabajador en un CEE, tomaríamos su salario anual $(8.436,33 €)$ y la aportación de la sociedad en subvenciones ( $\mathrm{I} 2.574,76 €$ ) obteniéndose un resultado de $0,6709 €$ o lo que es lo mismo, un porcentaje de retorno socioeconómico del $67,09 \%$.

Como hemos explicado anteriormente, para el cálculo con el total de la muestra, se ha realizado,

Tabla 4. Coste hora sociedad, y porcentaje de retorno socioeconómico del total de los 24 trabajadores de la muestra, en las dos modalidades de empleo

\begin{tabular}{|l|c|c|c|c|}
\hline & $\sum$ Coste neto anual sociedad & $\sum$ Horas año & Coste hora sociedad & $\bar{x}$ Coste hora sociedad \\
\hline ECA & $47.094,22 €$ & 33.072 & $1,42 €$ & $1,69 €$ \\
\hline CEE & $172.941,64 €$ & 33.072 & $5,23 €$ & $5,37 €$ \\
\hline & $\sum$ Salario neto anual & $\sum$ Subvenciones & $\begin{array}{c}\text { \% Retorno } \\
\text { socioeconómico }\end{array}$ & $\begin{array}{c}\bar{x} \% \text { Retorno } \\
\text { socioeconómico }\end{array}$ \\
\hline ECA & $304.968,54 €$ & $96.805,72 €$ & $315,03 \%$ & $348,60 \%$ \\
\hline CEE & $151.920,42 €$ & $182.732,23 €$ & $83,14 \%$ & $82,83 \%$ \\
\hline
\end{tabular}

Fuente: Elaboración propia. 
por un lado, un sumatorio anual de cada uno de los conceptos y la posterior división de los totales; y por otro lado, se ha calculado el dato para cada trabajador y se obtenido luego el dato medio. Podemos observar los resultados en la Tabla 4. Manejando los sumatorios, el coste hora sociedad de los trabajadores en ECA alcanza los I,42 $€$, mientras que al simular los contratos en CEE el coste neto hora asciende a $5,23 €$. Si calculamos los costes hora sociedad para cada trabajador y posteriormente la media de éstos, los resultados reflejan un coste neto sociedad de I,69 € en ECA y de 5,37 € en CEE. Paralelamente, manejando los sumatorios, el porcentaje de retorno socioeconómico en ECA asciende al 3 I $5,03 \%$, mientras que en el CEE apenas llega a un 83, I $4 \%$. Si calculamos el porcentaje de retorno socioeconómico para cada trabajador y posteriormente la media de éstos, obtenemos un $348,60 \%$ en ECA y un $82,83 \%$ en CEE.

\section{Discusión y conclusiones}

Lo primero que podemos decir a la luz de los datos obtenidos en este estudio de simulación y análisis coste-beneficio es que las PCD de nuestra muestra se benefician más en la simulación al trabajar en una modalidad de empleo lo más normalizada posible. Estos resultados se muestran coherentes con los de otros trabajos previos realizados fuera de España (Cimera, 2007a, 2007b, 2008, 201 2a).

Respecto al trabajador, si se compara el sueldo neto hora, podemos ver que en la situación de los trabajadores en empresa ordinaria mediante ECA se alcanzan 9,22 € frente a los 4,59€ que se alcanzarían en la simulación de los mismos trabajadores en CEE. Estas cifras son similares, pero algo más bajas, si utilizamos para comparar las medias: $9,03 €$ frente a $4,53 €$. Por tanto, el sueldo neto se duplica en la empresa ordinaria, es decir, un trabajador en ECA ganaría el doble que una situación similar en un CEE.

Respecto a la empresa, el coste neto hora es mucho más alto en la situación de empleo en empresa ordinaria ( $10,25 €)$ que en la simulada en CEE (०,93€). Las diferencias son bastante parecidas utilizando las medias, aunque el coste en CEE es aun menor, $9,85 €$ frente a $0,72 €$, respectivamente. Como es obvio, este resultado debe interpretarse en función del sistema de cálculo de la variable. Al calcular el coste neto anual de la empresa, se suman todos los costes y se resta a lo obtenido las bonificaciones y subvenciones. Por ello, al recibir los CEE una cantidad más elevada en términos de bonificaciones y subvenciones, el resultado de su coste neto es menor. Así entendido, la lectura que consideramos correcta es que un trabajador en un CEE supone menos coste para la empresa que uno en ECA, debido a la diferencia en subvenciones y bonificaciones recibidas a favor de los CEE. Esto se refleja de manera más clara cuando vemos el porcentaje de carga social, que pone en relación las bonificaciones y los gastos. En este caso, vemos cómo este porcentaje es sensiblemente más elevado en los $\operatorname{CEE}(85,54 \%)$ que en el ECA $(22,2 \mathrm{I} \%)$, con cifras muy semejantes, pero algo más altas al utilizar las medias, $89,74 \%$ y $23,99 \%$, respectivamente; ello se explica también en función de la mayor cantidad de subvenciones recibidas y del menor nivel de costes, en parte debido a exenciones. En algunos casos, al realizar la simulación situando al mismo trabajador en un hipotético contrato de similares características en un CEE, los costes para la empresa eran negativos, es decir, en términos netos, el CEE obtenía un beneficio simplemente por la contratación, una vez descontados los beneficios recibidos de los costes.

Con respecto a la sociedad, el aporte que ésta tiene que realizar para un contrato en un $\operatorname{CEE}(5,23 €)$ es casi cuatro veces superior $(\mathrm{I}, 42 €)$ al que debe realizar para contratar a una persona en empresa ordinaria mediante empleo con apoyo con una carga horaria similar. Las cifras son algo más altas al utilizar las medias para comparar, con $5,37 €$ y $\mathrm{I}, 69, €$ respectivamente. Esto significa que socialmente nos cuesta más insertar en CEE que en empresas ordinarias. Además, el porcentaje de retorno socioeconómico obtenido al poner en relación el salario neto y las subvenciones recibidas es casi cuatro veces más elevado en el empleo ordinario 
mediante ECA $(3$ I $5,03 \%)$ que en los CEE $(83, \mathrm{I} 4 \%)$, más de cuatro veces si comparamos las medias, con $348,60 \%$ y $82,83 \%$ respectivamente, por lo que podemos afirmar la mayor rentabilidad social del ECA en empresa ordinaria frente al empleo en CEE.

Conseguir un empleo es un objetivo fundamental para todas las personas, y no lo es menos entre las que tienen alguna discapacidad: todos tenemos como objetivo ser mejores profesionales, trabajar en una mejor posición y obtener el mayor beneficio posible por ello, algo que en la situación de crisis que vivimos en estos momentos cada vez se hace más complejo. Es por esto por lo que cobra una mayor importancia -tanto para la persona, la empresa como para la sociedad- disponer de una situación que aporte un mayor beneficio y los menores costes posibles.

Aunque con las debidas precauciones, para la muestra de trabajadores que forma parte de este estudio, y de acuerdo con la simulación, cuando se trabaja en empresa ordinaria mediante ECA se obtiene un mayor beneficio, pues llegan a cobrar casi el doble. Además, el porcentaje de carga social de la empresa es casi cuatro veces menor, y su rentabilidad social, cuatro veces superior. Resultados de otros estudios (Jordán de Urríes y Verdugo, 2010b y 20I2) muestran que los niveles de inversión por parte de la Administración son mucho más altos en el empleo protegido que en el empleo con apoyo. Los resultados indican que este hecho supone que se está invirtiendo un dinero que no se está recuperando adecuadamente en retornos para la sociedad, mientras que si se aumentase la inversión en empleo ordinario con apoyo, trabajador, empresa y sociedad se verían más beneficiados.

Como ya hemos afirmado, hemos de ser cautos al interpretar los datos obtenidos. En primer lugar, por el escaso tamaño de la muestra, ya que cualquier dato significativo necesitaría de una muestra amplia. Hay que tener en cuenta también que se han desconsiderado los costes de apoyo tanto del preparador en empleo con apoyo como de las unidades de apoyo a la actividad profesional en CEE. En segundo lugar, el tipo de estudio realizado, que simula situaciones semejantes del mismo trabajador de ECA en un CEE, hace que, en realidad, trabajemos sobre supuestos y no sobre datos reales. Además nuestra aproximación es de simple comparación de datos, sin análisis estadísticos en profundidad. Sin embargo, debemos tener en cuenta también la relevancia de haber seleccionado una muestra de trabajadores en empleo ordinario, es decir, que ya trabajan realmente integrados en empresas ordinarias, y compararla con situaciones hipotéticas en CEE, que tienen un marco mucho más acotado y concreto para poder extrapolar los datos. Esto además pone de manifiesto una realidad subyacente, que es la de que muchas personas que trabajan en CEE podrían y deberían estar en empresas ordinarias.

Los datos que nos aporta este estudio, a pesar de todo, pueden ser clarificadores y apuntan a los beneficios del ECA. Estos datos permiten recomendar la inclusión laboral de las PCD en empleo ordinario mediante empleo con apoyo frente a alternativas de empleo protegido, y señalar que se obtendría mayor beneficio para la persona, la empresa y la sociedad si la inversión de la Administración se orientase en este sentido. Sin embargo, aun asumiendo un marco internacional que determina la obligación de incluir a la persona en las modalidades de empleo más integradoras, podemos observar cómo en España no se desarrollado plenamente esta orientación, al subvencionarse sensiblemente más a los CEE que al ECA (Jordán de Urríes y Verdugo, 20IOb, 20II y 2OI2).

La valoración prudente que podemos extraer del presente trabajo es que debemos vislumbrar más allá de la barrera: la legislación apuesta por discriminar en positivo para que las personas con discapacidad puedan alcanzar la normalidad con los apoyos. Mientras tanto, seguimos encontrando ámbitos de segregación en el mundo del empleo. Debemos avanzar igual que lo hacen nuestros modelos, que nos marcan la obligatoriedad de integrar a la persona en todos los ámbitos comunitarios y dotarla de los apoyos oportunos para que pueda tener 
independencia y acceder a todos los recursos de la comunidad, ¿Por qué no se hace lo mismo con el empleo? Los que apuestan por el CEE, dirán que el fin de éste es conseguir que la persona pueda tener una remuneración y un trabajo digno, y en algunos casos añadirán que esto debe ser así mientras se preparan para acceder a las empresas ordinarias, pero esta vía de acceso no está funcionando, ya que las personas que se encuentran en CEE casi nunca pasan a trabajar en empleos integrados en la comunidad (Jordán de Urríes y Verdugo, 20Iob, 20I I y 20I2). Por todo ello, la sociedad, debe invertir y creer en el empleo integrado de las PCD, ofreciendo la oportunidad de trabajar en ECA y legislando a favor de la inclusión.

\section{Referencias bibliográficas}

Asociación Española de Empleo con Apoyo (2013): Informe contrataciones empleo con apoyo 2OI2. Madrid: Asociación Española de Empleo con Apoyo.

Beyer, S. (20I2): “The impact of agency organisation and natural support on supported employment outcomes". Journal of Vocational Rehabilitation, 36: ro9-I I9 (en línea). <http:// dx.doi.org/IO.3233/JVR-20I2-0586>.

Beyer, S. y Kilsby, M. (I998): "Financial costs and benefits of two supported employment agencies in Wales". Journal of Applied Research in Intellectual Disabilities, I I (4): 303-3 I9.

Beyer, S. et al., (I999): “The organisation and outcomes of supported employment in Britain”. Journal of Vocational Rehabilitation, I2: I37-I46.

Cimera, R.E. (2OI2a): "The economics of supported employment : What new data tell us". Journal of Vocational Rehabilitation, 37: I09-II7.

- (20I2b): "The outcomes achieved by previously placed supported employees with intellectual disabilities: Second verse same as the first ?". Journal of Vocational Rehabilitation, 36: 65-7I (en línea). <http://dx.doi.org/I0.3233/JVR2OI 2-0582>.

- (20IIa): "Does being in sheltered workshops improve the employment outcomes of supported employees with intellectual disabilities ?". Journal of Vocational Rehabilitation, 35: 2I-27 (en línea). <http:// dx.doi.org/I0.3233/JVR-20I I-0550>.

- (20I I b): "Supported versus sheltered employment : Cumulative costs, hours worked, and wages earned". Journal of Vocational Rehabilitation, 35: 85-92 (en línea). <http:// dx.doi.org/I0.3233/JVR-20I I-0556>.

- (2010): "The national cost-efficiency of supported employees with intellectual disabilities: The worker's perspective". Journal of Vocational Rehabilitation, 33: I 23-I3 I (en línea). <http://dx.doi.org/IO.3233/JVR-2OIO$052 \mathrm{I}>$.

- (2009a): "The national costs of supported employment to Vocational Rehabilitation: 2002 to 2006 ". Journal of Vocational Rehabilitation, 3०: I-9 (en línea). <http://dx.doi.org/Io.3233/ JVR-2009-0448>.

- (2009b): "The monetary benefits and costs of hiring supported employees: A pilot study". Journal of Vocational Rehabilitation, 30 : I I II 9 (en línea). <http://dx.doi.org/I0.3233/JVR2009-0457>.

- (2008): "The cost-trends of supported employment versus sheltered employment". Journal of Vocational Rehabilitation, 28: I 5-20. 
- (2007a): "The cost-effectiveness of supported employment and sheltered workshops in Wisconsin (FY 2002 - FY 2005)". Journal of Vocational Rehabilitation, 26: I 53 -I 58 .

- (2007b): "The cost of supported employment in Wisconsin (FY 2002 - FY 2005)". Journal of Vocational Rehabilitation, 26: 97-I04.

- (2006a): "The monetary benefits and costs of hiring supported employees: Revisited". Journal of Vocational Rehabilitation, 24: I37-I44.

- (2006b): "The future of supported employment: Don't panic!”. Journal of Vocational Rehabilitation, 24: I45-I49.

Cimera, R.E. y Burgess, S. (20II): “Do adults with autism benefit monetarily from working in their communities?". Journal of Vocational Rehabilitation, 34: I73-I80.

Egea, C. y Sarabia, A. (200I): "Clasificaciones de la OMS sobre discapacidad”. Boletín del Real Patronato sobre Discapacidad, 50: I 5-30.

EEUU. Rehabilitation Act Amendments of 1986, Pub. L. No. 99-506(I986).

España. Constitución Española. Boletín Oficial del Estado, núm. 3 I I, de 29 de diciembre de 1978 , pp. 29.3 I3-29.424.

- Ley I3/1982, de 7 de abril, de Integración Social del Minusválido. Boletín Oficial del Estado, núm. I03, de 30 de abril de I982, pp. II.IO6-II.II 2.

- Real Decreto 870/2007, de 2 de julio, por el que se regula el programa de empleo con apoyo como medida de fomento de empleo de personas con discapacidad en el mercado ordinario de trabajo. Boletín Oficial del Estado, núm. I68, de I4 de julio de 2007 , pp. 30.6I 8-30.622.

- Real Decreto Legislativo I/20r3, de 29 de noviembre, por el que se aprueba el Texto Refundido de la Ley General de derechos de las personas con discapacidad y de su inclusión social. Boletín Oficial del Estado, núm. 289, de 3 de diciembre de 20I3, pp. $95.635-95.673$.
Instituto Nacional de Estadística (20I4): Encuesta de Población Activa. Primer trimestre de 2014. Madrid: Instituto Nacional de Estadística.

- (20I I): El empleo de las personas con discapacidad. Explotación de la Encuesta de Población Activa y de la Base Estatal de Personas con Discapacidad. Madrid: Instituto Nacional de Estadística.

Jordán de Urríes, F.B. (20I I): “Acercamiento al empleo con apoyo y breve análisis de España e Iberoamérica”. Psicología, Conocimiento y Sociedad, 40 (4): 2I-40.

Jordán de Urríes, F.B. y Verdugo, M.Á. (20I2): "Situación de los centros especiales de empleo en España (II): aspectos valorativos”. Polibea, IO2: 43-50.

- (20II): "Situación de los centros especiales de empleo en España (I): impacto sobre el empleo". Polibea, гог: I 5-22.

- (20I0a): "El empleo integrado como meta irrenunciable”. Integra, I3 (37): I-I 2.

- (20Iob): Informe sobre la situación de los centros especiales de empleo en España. Salamanca: INICO.

Laloma, M. (2007): Empleo protegido en España. Análisis de la normativa legal y logros alcanzados. Madrid: Cinca.

Malo, M.A. (2003): Las personas con discapacidad en el mercado de trabajo español. Revista del Ministerio de Trabajo y Asuntos Sociales, 46: 99-I 26.

Maritza, G. (2005): "La nueva cultura de la discapacidad y los modelos de rehabilitación”. Aquichán, 5 (I): Io8-II 3.

Ministerio de Empleo y Seguridad Social (20I2): Guía laboral del Ministerio de Empleo y Seguridad Social, Madrid: Ministerio de Empleo y Seguridad Social (en línea). <http:// www.empleo.gob.es/es/Guia/>.

Ministerio de Sanidad y Política Social (2008): Estrategia global de acción para el empleo de personas con discapacidad 2008-20I2, Madrid: Ministerio de Trabajo, Inmigración, Sanidad y Política Social, 7-7I. 
Naciones Unidas. (2006): Convención sobre los Derechos de las Personas con Discapacidad y Protocolo Facultativo, Nueva York: Naciones Unidas.

Narrillos, H. (2OIO): "El SROI (social return on investment): un método para medir el impacto social de las inversiones”. Análisis Financiero, I I 3: 34-43.

Organización Internacional del Trabajo (2008): OIT Convenio sobre la readaptación profesional y el empleo (personas inválidas) núm. I 59, y la Recomendación núm. I68 / La Convención de las Naciones Unidas sobre los derechos de las personas con discapacidad, Ginebra: Organización Internacional del Trabajo, I-73.

Retolaza, J.L. (2012): Análisis coste-beneficio de las empresas de inserción: elaboración de un modelo de análisis costo-beneficio. Madrid: Editorial Académica Española.

Retolaza, J.L. y Ramos, M. (2005): “Modelización del costo de las empresas de inserción para la administración pública”. CIRIEC-España, Revista de Economía Pública, Social y Cooperativa”, 52:36I-385.

Rodríguez Álvarez, V. (20I 2): "Las políticas de empleo orientadas a personas con discapacidad: ¿evolución o retroceso?”. Revista del Ministerio de Empleo y Seguridad Social, го I: 77-96.

Rodríguez Álvarez, V. et al. (20I2): "Diferencias salariales por discapacidad y centros especiales de empleo". Cuadernos de Economía, 35 (98): IOO-II6.

Schalock, R.L. (I999): “Hacia una nueva concepción de la discapacidad", en Verdugo, M.Á. y Jordán de Urríes, F.B., Hacia una nueva concepción de la discapacidad, Salamanca: Amarú Ediciones, 79-I09.

Servicio Público de Empleo Estatal (20I2): Integración laboral de las personas con discapacidad. Madrid: Servicio Público de Empleo Estatal.

Shearn, J. et al. (2000): "The cost-effectiveness of supported employment for people with severe intellectual disabilities and high support needs: A pilot study". Journal of Applied Research in Intellectual Disabilities, I3: 2937 (en línea). <http://dx.doi.org/Io.I046 /j.I468-3I48.2000.00005.x>.

Verdugo, M.Á. et al., (2013): "Discapacidad e inclusión: derechos, apoyo y calidad de vida", en Verdugo, M.Á. y Schalock, R.L. (coords.), Discapacidad e inclusión. Manual para la docencia, Salamanca: Amarú Ediciones. 